ARTICLE

https://doi.org/10.1038/s41467-018-07994-1

\title{
Room temperature multi-phonon upconversion photoluminescence in monolayer semiconductor $\mathrm{WS}_{2}$
}

\author{
J. Jadczak¹, L. Bryja', J. Kutrowska-Girzycka (10 1, P. Kapuściński (iD ${ }^{1}$, M. Bieniek²,4, Y.-S. Huang ${ }^{3}$ \& P. Hawrylak ${ }^{2}$
}

Photon upconversion is an anti-Stokes process in which an absorption of a photon leads to a reemission of a photon at an energy higher than the excitation energy. The upconversion photoemission has been already demonstrated in rare earth atoms in glasses, semiconductor quantum wells, nanobelts, carbon nanotubes and atomically thin semiconductors. Here, we demonstrate a room temperature upconversion photoluminescence process in a monolayer semiconductor $\mathrm{WS}_{2}$, with energy gain up to $150 \mathrm{meV}$. We attribute this process to transitions involving trions and many phonons and free exciton complexes. These results are very promising for energy harvesting, laser refrigeration and optoelectronics at the nanoscale.

\footnotetext{
${ }^{1}$ Department of Experimental Physics, Wroclaw University of Science and Technology, Wroclaw 50-370, Poland. ${ }^{2}$ Department of Physics, University of Ottawa, Ottawa, K1N 6N5 Ontario, Canada. ${ }^{3}$ Department of Electronic Engineering, National Taiwan University of Science and Technology, Taipei 106, Taiwan. ${ }^{4}$ Department of Theoretical Physics, Wrocław University of Science and Technology, Wybrzeże Wyspiańskiego 27, 50-370 Wroclaw, Poland. Correspondence and requests for materials should be addressed to J.J. (email: joanna.jadczak@pwr.edu.pl)
} 
$\mathrm{P}$ hoton upconversion is an anti-Stokes process in which an absorption of a photon leads to a reemission of a photon at energy higher than the excitation energy. The upconversion photoemission has been already demonstrated in rare earth atoms in glasses ${ }^{1-3}$, semiconductor quantum wells ${ }^{4-7}$, nanobelts ${ }^{8}$, carbon nanotubes ${ }^{9}$ and atomically thin semiconductors ${ }^{10,11}$ at cryogenic temperatures. The atomically thin semiconductors based on transition metal dichalcogenides are particularly promising for room temperature upconversion due to their very strong photon-exciton ${ }^{12-32}$ and phonon-exciton interactions ${ }^{14}$.

Recently, Jones et al. ${ }^{10}$ reported phonon-mediated upconversion of photon emission by $\sim 30 \mathrm{meV}$ up to $250 \mathrm{~K}$, from trion $\left(\mathrm{X}^{-}\right)$ to exciton $(\mathrm{X})$, in a single layer of $\mathrm{WSe}_{2}$. This was possible because the strong confinement of carriers to a single layer results in a very high binding energy of an electron to an exciton forming a trion ${ }^{14-23,25}, \sim 30 \mathrm{meV}$, comparable with a phonon energy.

Here, we demonstrate a room temperature upconversion photoluminescence process in a monolayer semiconductor $\mathrm{WS}_{2}$ with a larger energy gain, up to $150 \mathrm{meV}$. We also show that the energy gain significantly depends on the temperature and increases from $42 \mathrm{meV}$ at $7 \mathrm{~K}$ to $150 \mathrm{meV}$ at $295 \mathrm{~K}$.

\section{Results}

Room temperature robust photon upconversion by $150 \mathrm{meV}$. Figure $1 \mathrm{a}-\mathrm{c}$ shows the main result of this work, demonstrating a robust upconversion of excited light. Figure 1a shows a schematic representation of our experiment. Photons from exciting laser field $E_{e x}$ couple the ground state to excited states with energy below the bandgap of a semiconductor ${ }^{7,9}$. Then the electrons in an excited state absorb phonons and are transferred to a higher energy excitonic state $\mathrm{X}$, from which they recombine to the ground state, emitting a photon at an energy higher than the exciting laser photon energy. Fig. 1b demonstrates upconversion of exciting laser photons in an atomically thin semiconductor $\mathrm{WS}_{2}$. A single layer of $\mathrm{WS}_{2}$ is deposited on a commonly used $\mathrm{SiO}_{2} / \mathrm{Si}$ substrate, and emission spectra are recorded in ambient environment and at room temperature of $295 \mathrm{~K}$. We excite with a laser having photon energy $\mathrm{E}_{\mathrm{ex}}=1.85 \mathrm{eV}$, indicated with an orange arrow, and observe emission at much higher energy, centred on a free exciton energy $\mathrm{E}_{\mathrm{X}} \approx 2.008 \mathrm{eV}$. In Figure $1 \mathrm{~b}$, we clearly see that the incident photons are converted into emitted photons with an energy higher by $150 \mathrm{meV}$, which is about five times more than previously observed in ref. ${ }^{10}$. For comparison, Fig. 1c demonstrates the effect of an extra hBN layer deposited between $\mathrm{SiO}_{2} / \mathrm{Si}$ and $\mathrm{WS}_{2}$ on the upconversion photoluminescence (UPC PL) process. It is clearly seen that exciting photons are also converted into emitted photons with energy higher by about $150 \mathrm{meV}$, even though the intensity of the upconversion emission detected in $\mathrm{WS}_{2} / \mathrm{hBN} / \mathrm{SiO}_{2} / \mathrm{Si}$ heterostructure is one order of magnitude lower than in $\mathrm{WS}_{2}$ deposited directly on the $\mathrm{SiO}_{2} / \mathrm{Si}$ substrate. The intensity of emitted upconverted photons in both $\mathrm{WS}_{2} / \mathrm{SiO}_{2} / \mathrm{Si}$ and $\mathrm{WS}_{2} / \mathrm{hBN} / \mathrm{SiO}_{2} / \mathrm{Si}$ heterostructures strongly correlates with the intensity of normal photoluminescence (PL), spectral shape of which strongly depends on the thickness of hBN layer. This is why, for further studies of the excitonic upconversion photoluminescence in ambient at $295 \mathrm{~K}$, we choose single layers of $\mathrm{WS}_{2}$ exfoliated on $\mathrm{SiO}_{2} / \mathrm{Si}$ substrate, as they exhibit the most prominent emission attributed to the neutral exciton (X).

Let us now fully characterize the upconversion photoluminescence process, i.e., variations in the upconverted photoemission spectrum as a function of an incident photon energy $\mathrm{E}_{\mathrm{ex}}$. In this experiment, a short-pass edge filter (EF), with the edge at $632 \mathrm{~nm}(1.97 \mathrm{eV})$, was used. For all exciting photon energies $\mathrm{E}_{\mathrm{ex}}$ one line at $\sim 2.008 \mathrm{eV}$, related to the neutral exciton $\mathrm{X}$ emission, is observed in the spectra. The upconversion photoluminescence intensity of this line strongly depends on the excitation photon energy, whereas spectral shape and peak position remain nearly unchanged upon variation of the excitation energy, which is clearly seen in Fig. 2a. Figure 2a shows the colour map of the upconversion photoemission intensity as a function of the exciting photon energy. The exciting photon energy required to achieve a detectable upconversion photoluminescence (gradual intensity growth just above the noise floor) is found to exceed $\mathrm{E}_{\mathrm{ex}}=1.85 \mathrm{eV}$. Fig. $2 \mathrm{~b}$ presents the dependence of the upconversion photoluminescence integrated intensity in the spectral range from $1.91 \mathrm{eV}$ to $2.10 \mathrm{eV}$ on the energy difference $\left(\mathrm{E}_{\mathrm{X}}-\mathrm{E}_{\mathrm{ex}}\right)$ of the exciton $\mathrm{X}$ and the exciting photon energy $\mathrm{E}_{\mathrm{ex}}$. For the energy difference $E_{X}-E_{e x}$ from $50 \mathrm{meV}$ to $155 \mathrm{meV}$, the integrated upconversion photoluminescence intensity decreases with decreasing excitation photon energy with the rate equal to $-0.0256 \mathrm{meV}^{-1}$.
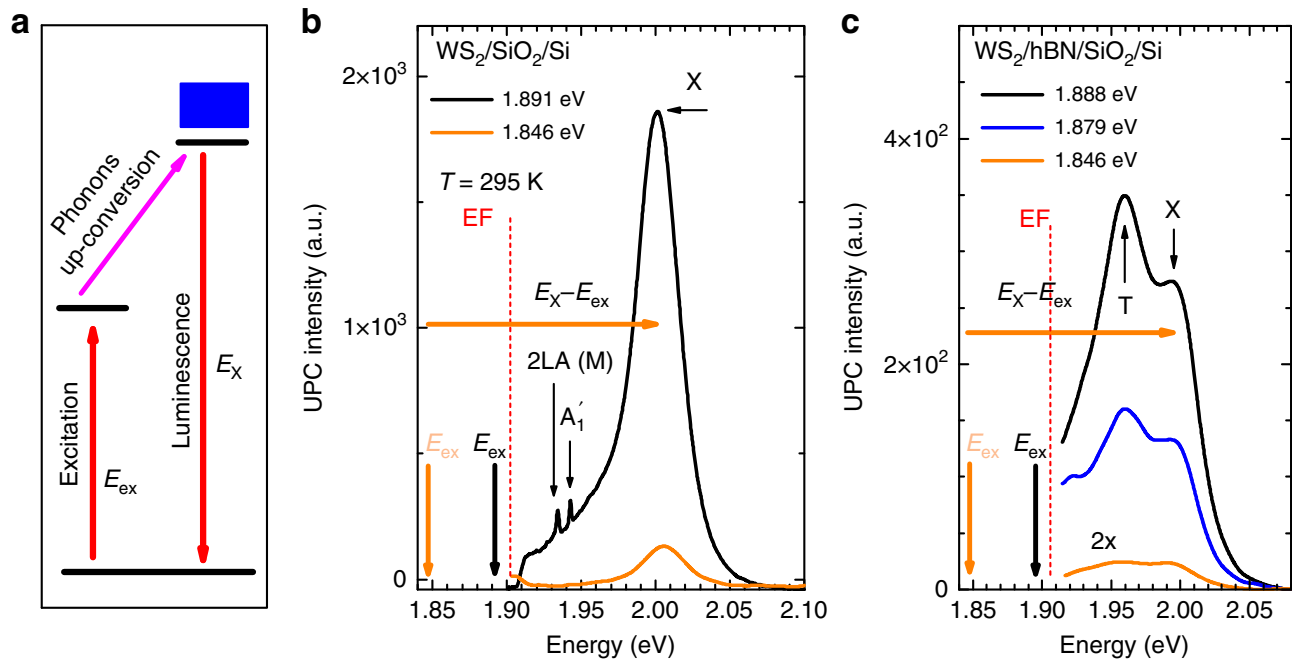

Fig. 1 Upconversion photoemission process in monolayer $\mathrm{WS}_{2}$. a The schematic representation of upconversion process. $\mathbf{b}$ Examples of the upconversion photoemission spectra in $\mathrm{WS}_{2} / \mathrm{SiO}_{2} / \mathrm{Si}$ recorded for different excitation photon energies. c Examples of the upconversion photoemission spectra in WS $\mathrm{W}_{2} /$ $\mathrm{hBN}(136 \mathrm{~nm}) / \mathrm{SiO}_{2} / \mathrm{Si}$ detected for different excitation photon energies. To reduce laser scattering light short-pass (in wavelength) edge filters (EF) with edge at $652 \mathrm{~nm}(1.90 \mathrm{eV})$ were used 
a

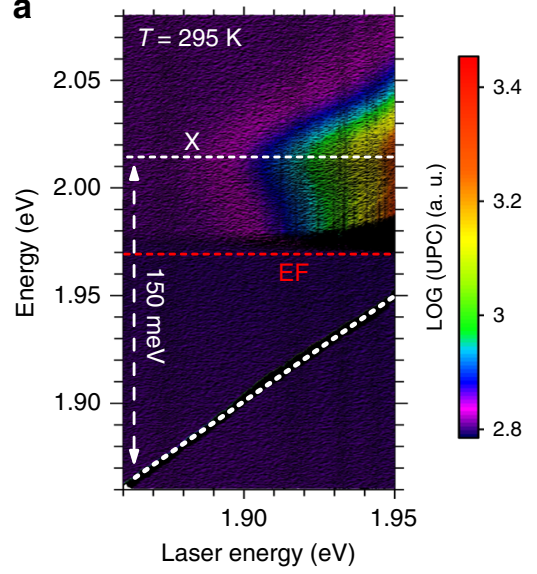

b

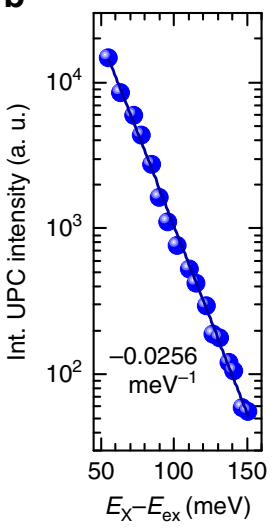

Fig. 2 Excitation energy dependence of the upconversion photoemission. a Evolution of the upconversion emission spectra as a function of the laser excitation photon energy in $\mathrm{WS}_{2} / \mathrm{SiO}_{2} / \mathrm{Si}$, recorded in ambient, at $295 \mathrm{~K}$. b The dependence of the upconversion integrated intensity on the energy difference of the neutral exciton $(X)$ and the excitation photon energy $\left(E_{X}-E_{e x}\right)$

Identifying the upconversion photoluminescence mechanism. We now turn to identifying mechanism responsible for the upconversion photoluminescence. Figure $3 \mathrm{a}$ shows photoluminescence spectra excited with laser having photon energy of $2.33 \mathrm{eV}(532 \mathrm{~nm})$. The spectra were measured in ambient at $295 \mathrm{~K}$ for monolayer $\mathrm{WS}_{2}$ deposited on the $\mathrm{SiO}_{2} / \mathrm{Si}$ substrate or hBN/ $\mathrm{SiO}_{2} / \mathrm{Si}$ substrates with different thicknesses of hBN layers (21 $\mathrm{nm}, 136 \mathrm{~nm}, 342 \mathrm{~nm}$ ), respectively. In the PL spectrum of the $\mathrm{WS}_{2} / \mathrm{SiO}_{2} / \mathrm{Si}$ (blue line), we distinguish only one, dominant, spectral line at $2.014 \mathrm{eV}$. In comparison with previous studies, including ours ${ }^{17-21}$, we attribute this line to the free exciton $\mathrm{X}$ emission. For all the $\mathrm{WS}_{2} / \mathrm{hBN} / \mathrm{SiO}_{2} / \mathrm{Si}$ heterostructures with finite $\mathrm{hBN}$ thickness, we observe $\mathrm{X}$ line redshifting to $2.006 \mathrm{eV}$, about $8 \mathrm{meV}$ below its position recorded for the $\mathrm{WS}_{2}$ monolayer deposited on $\mathrm{SiO}_{2} / \mathrm{Si}$. This energy difference is related to the change of the exciton-binding energy $\mathrm{E}_{\mathrm{b}}$ and the bandgap energy $\mathrm{E}_{\mathrm{g}}\left(\mathrm{E}_{\mathrm{X}}=\mathrm{E}_{\mathrm{g}}-\mathrm{E}_{\mathrm{b}}\right)$. The different dielectric environments of $\mathrm{WS}_{2}$ monolayers lead to the reduction of both $\mathrm{E}_{\mathrm{g}}$ and $\mathrm{E}_{\mathrm{b}}$ energies in $\mathrm{WS}_{2} / \mathrm{hBN} / \mathrm{SiO}_{2} / \mathrm{Si}$ heterostructures in comparison with the $\mathrm{WS}_{2} /$ $\mathrm{SiO}_{2} / \mathrm{Si}$ structures. Moreover, additional emission lines appear in the emission spectra of $\mathrm{WS}_{2} / \mathrm{hBN} / \mathrm{SiO}_{2} / \mathrm{Si}$ structures. One of the lines, positioned at the energy of $1.964 \mathrm{eV}, 42 \mathrm{meV}$ below that of the exciton $(\mathrm{X})$, is attributed to a negatively charged exciton, trion (T), whereas the lower-energy broad features labelled as $\mathrm{L}$, positioned at $\sim 1.86 \mathrm{meV}$, are attributed to strongly localized excitons ${ }^{20,21}$. The $\mathrm{L}$ lines in the $\mathrm{PL}$ of the $\mathrm{WS}_{2} / \mathrm{hBN} / \mathrm{SiO}_{2} / \mathrm{Si}$ heterostructures are recorded about $150 \mathrm{meV}$ below that of the free exciton. These results show that an extra hBN layer used between the flake and $\mathrm{SiO}_{2} / \mathrm{Si}$ substrate can act as a buffer layer ${ }^{33}$ and changes the doping level in the monolayer system. Here, it is manifested by the altering trion to exciton emission intensity ratio $(\mathrm{T} / \mathrm{X})$ shown in the inset of Fig. 3a. The $\mathrm{X}$ and $\mathrm{T}$ emission intensities are obtained by the fitting of the PL spectra by Lorentz function in spectral range from $1.7 \mathrm{eV}$ to $2.10 \mathrm{eV}$. For normal $\mathrm{WS}_{2} / \mathrm{SiO}_{2} / \mathrm{Si}$ structure, the trion peak is hardly detected in the PL spectra, whereas for $\mathrm{WS}_{2} / \mathrm{hBN} / \mathrm{SiO}_{2} / \mathrm{Si}$ heterostructures with increasing $\mathrm{hBN}$ thicknesses the T/X PL intensity ratio increases. The rising value of the T/X PL intensity ratio indicates that the increasing of hBN layer thickness leads to the growth of twodimensional electron gas concentration in monolayer $\mathrm{WS}_{2}$. This dependence can be explained by the fact that the $\mathrm{WS}_{2}$ is naturally n-doped whereas positively charged defects (or charge inhomogeneity) are embedded in $\mathrm{SiO}_{2}$ surface, close to the monolayer $\mathrm{WS}_{2}$. Hence, the positively charge defects in $\mathrm{SiO}_{2}$ surface strongly influence an electron charge in $\mathrm{WS}_{2}$. Intuitively, the electron-binding energy to the charged defect embedded in $\mathrm{SiO}_{2}$ is the largest for $\mathrm{WS}_{2} / \mathrm{SiO}_{2} / \mathrm{Si}$ structures and should decrease with increasing thickness of hBN layer. Our experimental results are qualitatively consistent with recent theoretical calculations of the ground-state energies of electrons as a function of the distance of a positive point-charge defect from the mid-plane of the monolayer ${ }^{34}$. Namely, Tuan et al. ${ }^{34}$ have shown that the electronbinding energy is the largest when the defect is at the surface, and it significantly decreases from $150 \mathrm{meV}$ to a few $\mathrm{meV}$ as the distance between point-charge defect and the mid-plane of the monolayer exceeds $100 \mathrm{~nm}$. Interestingly, the $\mathrm{L}$ lines attributed to localized excitons emerge in the PL spectra on the low energy wing of the trion when hBN layer is placed between the $\mathrm{WS}_{2}$ monolayer and $\mathrm{SiO}_{2}$ substrate and they are well resolved in the spectra and comparable with the trion emission for hBN thickness higher than $300 \mathrm{~nm}$ (Fig. 3a). when the contact with extrinsic defects in $\mathrm{SiO}_{2}$ is reduced.

In order to get further inside into the nature of observed excitonic complexes, we perform complementary temperaturedependent PL measurements. Figure $3 \mathrm{~b}$ compares low temperature $(7 \mathrm{~K})$ spectra, normalized to maximum photoluminescence intensity, detected for selected $\mathrm{WS}_{2} / \mathrm{hBN} / \mathrm{SiO}_{2} / \mathrm{Si}$ heterostructures with different thicknesses of hBN layers $(21 \mathrm{~nm}, 120 \mathrm{~nm}, 136$ $\mathrm{nm})$, respectively. There is a qualitative correlation between the energy of the peak associated with particular transition and the thickness of the hBN layer. The emission lines detected at higher energies, labelled as $\mathrm{X}, \mathrm{T}_{\mathrm{S}}, \mathrm{T}_{\mathrm{T}}$ and $\mathrm{L}_{0}$, exhibit an apparent red shift with increasing thickness of hBN (for X line see Fig. 3c), whereas the series of lower energy $\mathrm{L}$ lines remains energetically inert. Based on this observation, we divide all observed optical transitions into two groups. The first group is associated with the nearly free states of the neutral exciton (X), intravalley spin singlet trion $\left(T_{S}\right)^{10,19-21}$, intervalley spin triplet trion $\left(T_{T}\right)^{10,19-21}$ and intervalley singlet trion $\left(\mathrm{L}_{0}\right)^{20,21}$, respectively. The second group is attributed to the strongly localized states, likely excitons bound to donors or acceptors. Donor or acceptor levels likely originate from vacancies, which are randomly created during mechanical exfoliation, therefore the emission intensity of bound excitons differs slightly from flake to flake due to different amount and types of created vacancies and the difference in the local two-dimensional electron gas concentration. We have observed the effects related to inhomogeneous two- dimensional electron gas concentration in monolayer $\mathrm{WS}_{2}$ in our previous studies $^{21}$, where we have found a correlation between the strength of the trion and exciton resonances in $\mathrm{RC}$ spectra recorded at different points on the monolayer. Moreover, the $\mathrm{L}$ states are strongly localized in the gap, hence they are weakly influenced by the change of the dielectric environment and remain energetically inert. These optical transitions also do not play a significant role in absorption type experiments, such as reflectance contrast (RC) spectroscopy. Figure $3 \mathrm{~d}$ compares the photoluminescence and reflectivity contrast spectra recorded at $\mathrm{T}=7 \mathrm{~K}$ for the $\mathrm{WS}_{2} / \mathrm{hBN}$ $(21 \mathrm{~nm}) / \mathrm{SiO}_{2} / \mathrm{Si}$ structure. It is clearly seen that in the reflectivity contrast measurements, we probe only nearly free states $\left(X, T_{T}\right.$, $\mathrm{T}_{\mathrm{S}}$ and $\mathrm{L}_{0}$ ), whereas $\mathrm{L}$ series is not detected. On the other hand, the L peaks are clearly seen in the PL spectra. The reason for the difference in the strength of optical amplitude of the different excitonic transitions in PL and RC spectra is that the strength of excitons resonances in reflectivity is determined by respective density of states, whereas the PL intensity is contributed additionally by a state occupation factor.

Finally, let us analyse the temperature evolution of photoluminescence spectra detected for $\mathrm{WS}_{2} / \mathrm{hBN}(21 \mathrm{~nm}) / \mathrm{SiO}_{2} / \mathrm{Si}$ 

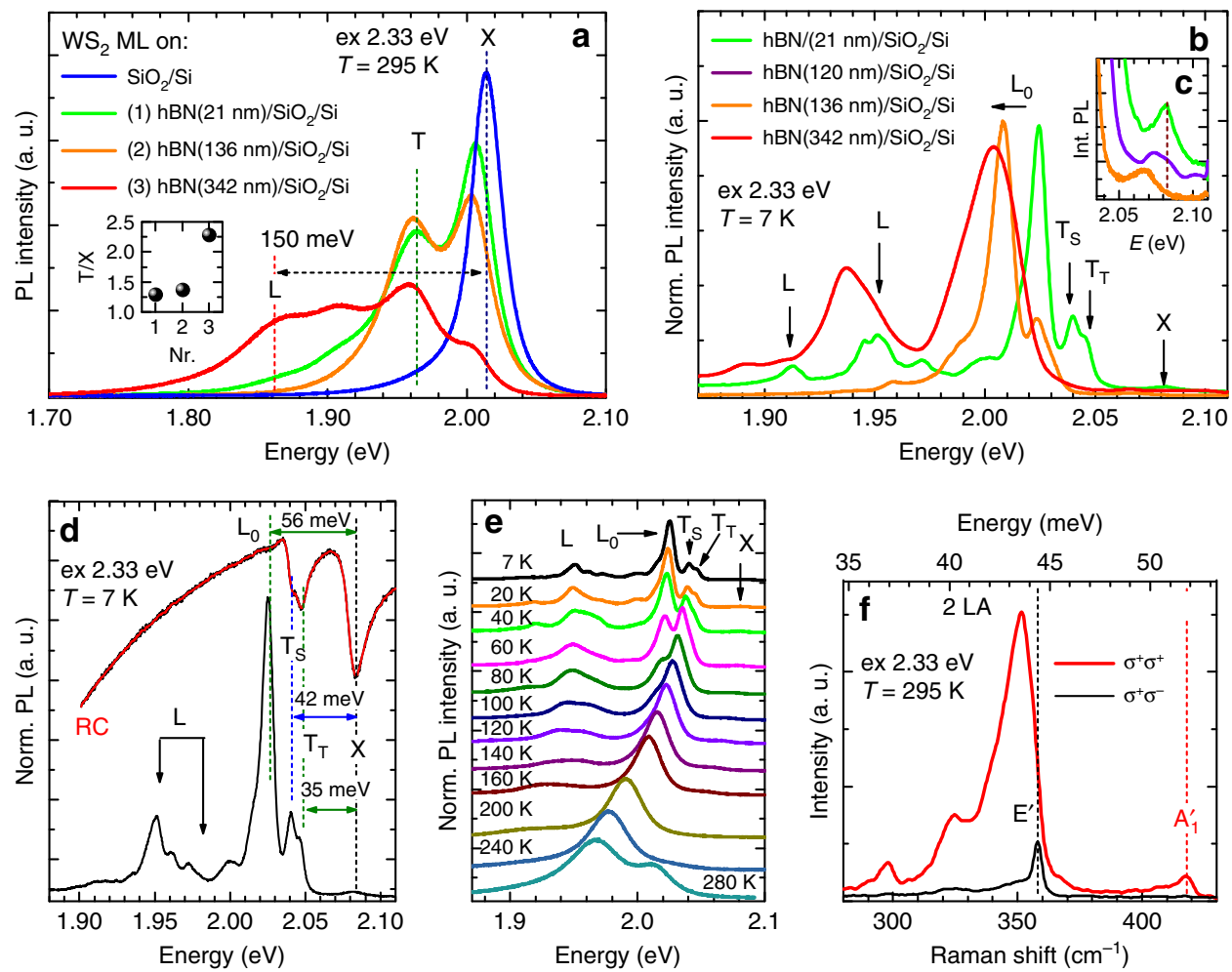

Fig. 3 Photoluminescence, reflectance contrast and Raman scattering. a Examples of photoluminescence spectra of monolayer $\mathrm{WS}_{2}$ deposited on $\mathrm{SiO}_{2} / \mathrm{Si}$ substrate and on $\mathrm{hBN} / \mathrm{SiO}_{2} / \mathrm{Si}$ substrates with different thicknesses of hBN layers, excited with incident photon energies of $2.33 \mathrm{eV}$ ( $532 \mathrm{~nm}$ ), and recorded in ambient at $295 \mathrm{~K}$. The inset shows the T/X emission intensity ratio for different flakes, 1, 2 and 3, respectively. b Examples of photoluminescence spectra of monolayer $\mathrm{WS}_{2}$ deposited on $\mathrm{hBN} / \mathrm{SiO}_{2} / \mathrm{Si}$ substrates with different thicknesses of hBN layers, excited with incident photon energies of $2.33 \mathrm{eV}(532 \mathrm{~nm})$, and recorded in vacuum at $7 \mathrm{~K}$. c The red-shift of neutral exciton $(X)$ emission line for increasing thickness of hBN layer. d The comparison of low temperature $\mathrm{PL}$ and $\mathrm{RC}$ spectra recorded in $\mathrm{WS}_{2} / \mathrm{hBN}(21 \mathrm{~nm}) / \mathrm{SiO}_{2} / \mathrm{Si}$. e The temperature evolution of $\mathrm{PL}$ spectra in WS $/ \mathrm{hBN}$ $(21 \mathrm{~nm}) / \mathrm{SiO}_{2} / \mathrm{Si}$. f Helicity resolved Raman spectra excited with $532 \mathrm{~nm}$ laser line for $\mathrm{WS}_{2} / \mathrm{SiO}_{2} / \mathrm{Si}$

structure, which are presented in Fig. 3e. We see that the L series significantly decays above $160 \mathrm{~K}$. It can be explained by the fact that the excitons gain kinetic energy and become less susceptible to the capture process. For different $\mathrm{WS}_{2} / \mathrm{hBN} / \mathrm{SiO}_{2} / \mathrm{Si}$ samples (not presented here), this effect occurs for slightly higher or lower temperatures and depends on the thickness of the hBN layer. Also, we observe that the energy position of the L series does not change as clearly as for nearly free states, which rapidly approach localized exciton energies with increasing temperature.

We now turn to identifying potential phonon modes involved in the upconversion process. Figure $3 \mathrm{f}$ presents helicity resolved Raman scattering spectra of a monolayer $\mathrm{WS}_{2}$ deposited on an $\mathrm{SiO}_{2} / \mathrm{Si}$ substrate excited with the $532 \mathrm{~nm}$ laser line with $\sigma^{+}$ polarized excitation and detection either in $\sigma^{+}$or $\sigma^{-}$polarization, labelled $\sigma^{+} \sigma^{+}$or $\sigma^{+} \sigma^{-}$. This method allows us to resolve and precisely assign degenerate Raman bands for the monolayer $\mathrm{WS}_{2}{ }^{35}$. The first order out-of-plane $A_{1}^{\prime}$ mode is visible only in $\sigma^{+} \sigma^{+}$configuration, whereas the in-plane E' is detected only in the spectra of opposite helicity $\sigma^{+} \sigma^{-}$. The Raman spectra clearly identify the $\mathrm{E}^{\prime}$ and $\mathrm{A}_{1}{ }_{1}$ optical phonons with energies $\sim 44 \mathrm{meV}$ $\left(357 \mathrm{~cm}^{-1}\right)$ and $\sim 52 \mathrm{meV}\left(418 \mathrm{~cm}^{-1}\right)$, respectively. In the upconversion photoluminescence process, the $\mathrm{A}_{2}$ " vibration at $441 \mathrm{~cm}^{-1}(\sim 55 \mathrm{meV})^{36}$ can be also considered. This phonon is Raman inactive in bulk, but becomes partially active for one or a few layers under resonant excitation ${ }^{37}$.

Jones et al. ${ }^{10}$ have attributed the upconversion photoluminescence process from a negatively charged exciton to a neutral exciton resonance in monolayer $\mathrm{WSe}_{2}$, producing spontaneous anti-Stokes emission with an energy gain of $30 \mathrm{meV}$, to doubly resonant Raman scattering mediated by the $\mathrm{A}_{1}$ optical phonon.
They based their interpretation on detailed polarization resolved experiments and the fact that the $30 \mathrm{meV}$ separation between neutral and charged excitons coincides with the $\mathrm{A}_{1}{ }_{1}$ energy equal to $31 \mathrm{meV}$. They observed preservation of valley polarization for excitation both above and below $\mathrm{X}$, consistent with the polarization properties of the $A_{1}^{\prime}$ phonon. In contrast, the $E^{\prime}$ mode in Raman spectra from $\mathrm{WSe}_{2}$, is cross-polarized and unpolarized for circularly and linearly polarized excitation, respectively. Additionally, the symmetry of the electronic states within the $\mathrm{K}$ valleys dictates a stronger interaction with the outof-plane $\mathrm{A}_{1}^{\prime}$ phonon than with the in-plane $\mathrm{E}^{\prime}$ mode ${ }^{38,39}$.

We observe the same (as in ref. ${ }^{10}$ ) polarization-dependent relation between the normal and upconversion photoluminescence and the $\mathrm{A}_{1}^{\prime}$ optical phonon in Raman spectra. In contrast to ref. ${ }^{10}$, where the upconversion photoluminescence process is mediated by one $A_{1}^{\prime}$ phonon, in our experiment more phonons are involved in the upconversion process. At room temperature the energy gain of $150 \mathrm{meV}$ in the upconversion photoluminescence process in monolayer $\mathrm{WS}_{2}$ closely matches the energy of three $A_{1}^{\prime}$ phonons equals to $156 \mathrm{meV}$. While, the three $A_{1}^{\prime}$ phonon-mediated upconversion of photon emission by $150 \mathrm{meV}$ is most probable, other combinations of optical phonons, $A_{1}{ }_{1}, E^{\prime}$ and $\mathrm{A}_{2}{ }^{\prime \prime}$, in the observed upconversion photoluminescence process should be also considered. For example, in the recent work Tuan et al. ${ }^{34}$ have proposed a model of phonon-assisted recombination facilitated by virtual trion states.

What is the origin of the initial states in the upconversion photoluminescence process? One of the possible explanation is that incident photon is in resonance with an exciton localized on impurity. This assignment of the initial upconversion state seems 
to be reasonable due to the proximity of localized states with the lowest excitation energies $(\sim 150 \mathrm{meV})$. However, in pseudoabsorption reflectivity contrast experiment we do not observe any features at energies specific to excitons localized on impurities. While this could be explained due to significant distribution of different impurity states, absence of any features as observed in, e.g., L band, shows that the absorption on excitons localized on impurities is week. In contrast, we observe the exponential drop of upconversion intensity as excitation energy is lowered from the exciton into the energy gap (see Fig. 2). Hence, similarly as in ref. ${ }^{10}$, we attribute the observed upconversion photoluminescence to the spontaneous excitonic anti-Stokes Raman scattering process, where the incident and emitting photons are in resonance with the trion and neutral exciton, respectively.

In contrast to the observations of Jones et al. ${ }^{10}$ where in the upconversion photoluminescence from the charged to the neutral exciton one phonon was involved, in our experiments the upconversion is related to multi-phonon process. The absorption of an incident photons is related to the states from the tail of the trion (see Fig. 3a). Moreover, in our previous studies ${ }^{21}$ we have observed that the absorption features broaden significantly as temperature increases, which leads to the increase of the absorption from the trion tail. Also, as it will be shown below, we detect the maximum of upconversion intensity for the excitation energy equal to the trion energy. Further experimental and theoretical studies are planned to elucidate the upconversion mechanism.

Power and carrier density dependence of upconversion spectrum. To gain more insight into the character of the excitonic upconversion photoluminescence in monolayer $\mathrm{WS}_{2}$, we performed excitation power-dependent measurements, with the excitation photon energy of $1.890 \mathrm{eV}$, corresponding to the broad emission of the localized excitons L (see Fig. 4). The experiments were carried out on the $\mathrm{WS}_{2}$ monolayer deposited on $\mathrm{SiO}_{2} / \mathrm{Si}$ at room temperature, in both ambient and vacuum conditions. The two different conditions allow us to investigate carrier density dependence of the upconversion process. It is well established that under ambient conditions the physisorbed $\mathrm{O}_{2}$ and $\mathrm{H}_{2} \mathrm{O}$ molecules deplete n-type materials such as $\mathrm{WS}_{2}$ or $\mathrm{MoS}_{2}$, much more than conventional electric field gating 22,23 . This is also clearly manifested in Fig. 4a, where the spectral shape of lines strongly differs between experiments performed in ambient (black line) and vacuum (red lines). This confirms previous reports that in ambient, the two-dimensional electron gas (2DEG) concentration is strongly depleted ${ }^{21}$. The upconversion photoluminescence spectra of $\mathrm{WS}_{2}$ measured in ambient conditions are dominated by the exciton emission $\mathrm{X}$, whereas the trion $\mathrm{T}$ is hardly detected in the spectra. In contrast, in vacuum the upconversion photoluminescence spectra clearly resolves emission of both the exciton and trion with comparable intensities. Their energy separation equals to $42 \mathrm{meV}$, and matches the values reported in previous studies ${ }^{20,21}$. Additionally, Fig. $4 \mathrm{~b}$ shows the comparison of the anti-Stokes Raman spectra measured in ambient (black line) and vacuum (red line), obtained by subtracting a Lorentz fit of the upconversion emission from the total upconversion emission spectrum (Fig. 4a), where the Raman features are indicated by the dashed rectangle. The Raman scattering peaks seen in ambient at $352 \mathrm{~cm}^{-1}$ and at $418 \mathrm{~cm}^{-1}$ are assigned to the 2 LA (M) and $A_{1}^{\prime}$ phonon modes, respectively. In vacuum, these phonons shift towards lower frequencies by $5 \mathrm{~cm}^{-1}$ and $2 \mathrm{~cm}^{-1}$, respectively. Moreover, in ambient, in the highest energy region we can also distinguish weak broad features at about $446 \mathrm{~cm}^{-1}(\sim 55 \mathrm{meV}), 470 \mathrm{~cm}^{-1}$ and $495 \mathrm{~cm}^{-1}$, similarly to work of Molas et al. ${ }^{40}$, who report resonant Raman scattering study of monolayer $\mathrm{WS}_{2}$ and propose identification of the peaks at $470 \mathrm{~cm}^{-1}$ and $495 \mathrm{~cm}^{-1}$ as the combined acoustic processes; $\mathrm{LA}(\mathrm{M})+2 \mathrm{ZA}(\mathrm{M})$ and $2 \mathrm{LA}(\mathrm{M})+\mathrm{ZA}(\mathrm{M})$, respectively. The
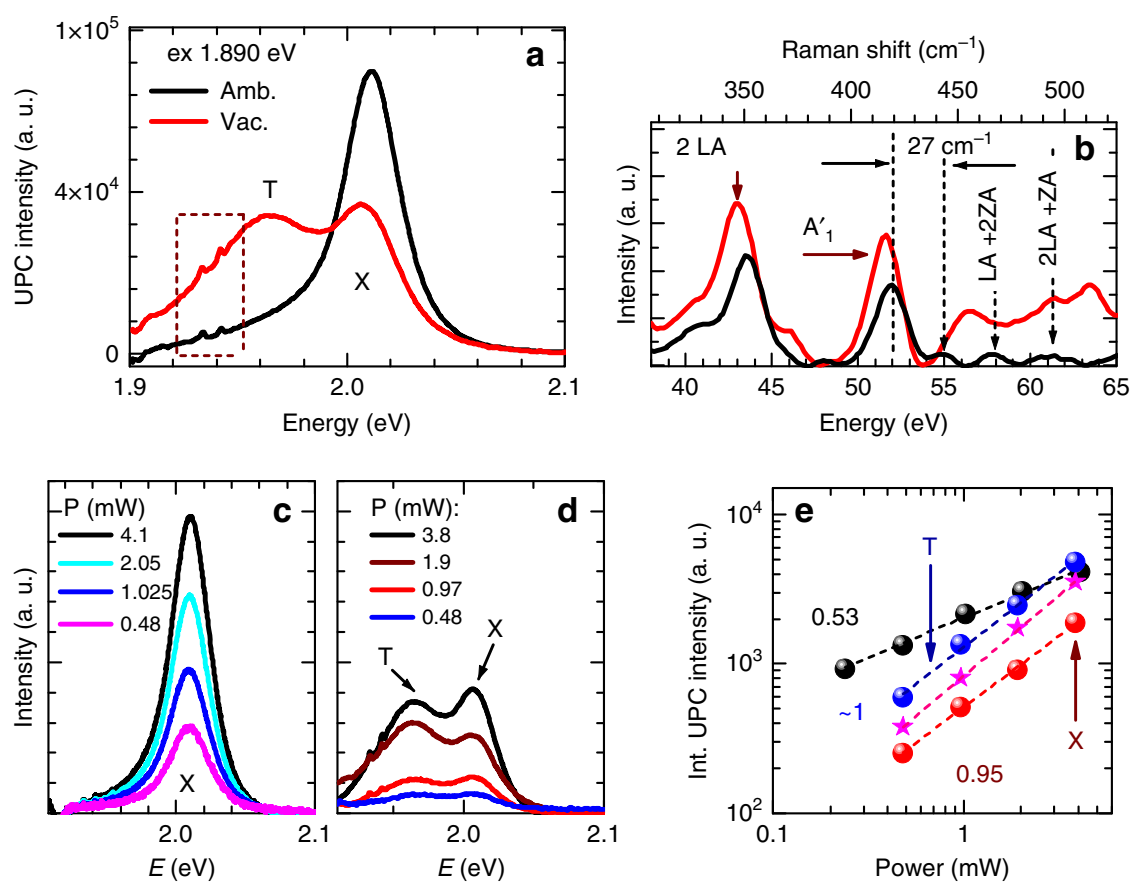

Fig. 4 Excitation power dependence of the upconversion photoemission. a Comparison of the spectra in monolayer $\mathrm{WS}_{2}$ deposited on $\mathrm{SiO}_{2} / \mathrm{Si}_{\mathrm{s}}$ substrate, recorded in ambient (black line) and vacuum (red line), excited with the same incident photon energy 1.890 eV and power, at $295 \mathrm{~K}$. b Comparison of the anti-Stokes Raman spectra measured in ambient (black line) and vacuum (red line), obtained by subtracting the emission from the corresponding UPC spectra in a. c, d examples of spectra excited with different laser powers, recorded in ambient and vacuum, respectively. e The integrated upconversion photoemission intensity plotted as a function of excitation power density for total spectra recorded in ambient (black circles), in vacuum (magenta stars), for $X$ peak in vacuum (red points), for $T$ peak in vacuum (blue point) 
feature observed in our experiment at the energy $\sim 27 \mathrm{~cm}^{-1}$ higher than the energy of the $A_{1}^{\prime}$ peak may be assigned to the partially active $\mathrm{A}_{2}$ phonon or it can result from multi-phonon processes, which involve principal optical phonons (E' or A') and additional acoustic phonons ${ }^{40}$.

Figure $4 \mathrm{c}$ and $\mathrm{d}$ presents typical photoemission spectra recorded for several excitation power densities, in ambient and in vacuum, respectively. Figure $4 \mathrm{e}$ shows the total upconversion intensity in ambient (black points) and vacuum (magenta stars) as a function of excitation power density, integrated over the same energy range from $1.9 \mathrm{eV}$ to $2.1 \mathrm{eV}$. They reveal weak sublinear (0.53) and linear ( 1) dependence for increasing excitation power in ambient and in vacuum, respectively. In addition, Fig. 4e presents power dependence of the $\mathrm{X}$ and $\mathrm{T}$ emission intensities obtained by fitting of the upconversion emission spectrum by Lorentz function in corresponding spectral range $(1.9-2.1 \mathrm{eV})$. The $\mathrm{X}$ (red points) and $\mathrm{T}$ (blue points) emissions show nearly linear $(0.95)$ and linear $(\sim 1)$ dependence, respectively. Interestingly, our observation clearly shows that upconversion mechanism strongly depends on 2DEG concentration. The weak sublinear dependence in ambient may be related to the changes of the charge on donor states 25,41 .

Nevertheless, we assume that both mechanisms originate from the multiple-phonon-assisted upconversion, since the threshold energy gain of $\sim 150 \mathrm{meV}$ indicates that the upconversion can be related to the Raman scattering process involving particular combination of the optical phonons. Moreover, for our experimental conditions, photons with the energy below the gap are sufficient to photo-ionize electrons from the donor level, which according to recent numerical calculation is positioned a few hundred meV below the conduction band ${ }^{25}$. Furthermore, as all the data of total upconversion intensities follow the sublinear and linear relationship, we exclude the possibility of nonlinear optical generation of the observed upconversion photoluminescence, such as two-photon excitation-induced emission ${ }^{42,43}$ and exciton Auger scattering ${ }^{44,45}$.

Temperature dependence of the upconversion photoluminescence. It is important that the upconversion photoemission observed here is detected at room temperature. In order to gain further insight into the temperature dependence of the mechanism of the upconversion emission, we carried out temperature-dependent measurements of both the normal and upconverted photoluminescence of the $\mathrm{WS}_{2} / \mathrm{SiO}_{2} / \mathrm{Si}$ and $\mathrm{WS}_{2} /$ $\mathrm{hBN} / \mathrm{SiO}_{2} / \mathrm{Si}$ structures. The normal photoluminescence is excited at all temperatures with the same photon energy equals to $2.33 \mathrm{meV}$. The excitation energy of the upconversion photoluminescence is tuned to observe a detectable emission, and is varying from $1.959 \mathrm{eV}$ to $2.033 \mathrm{eV}$ at particular temperatures. Figure $5 \mathrm{a}$ shows a PL spectrum measured at $7 \mathrm{~K}$ for the $\mathrm{WS}_{2} / \mathrm{hBN}$ $(136 \mathrm{~nm}) / \mathrm{SiO}_{2} / \mathrm{Si}$ structure. The coloured arrows indicate excitation energies $\left(\mathrm{E}_{\mathrm{ex}}\right)$ of the upconversion photoluminescence presented in the Fig. $5 \mathrm{~b}$. The exciting photon energy required to achieve a detectable upconversion photoluminescence (gradual intensity growth just above the noise floor) at $7 \mathrm{~K}$ is found to exceed $\mathrm{E}_{\mathrm{ex}}=2.022 \mathrm{eV}$. Figure $5 \mathrm{c}$ shows the dependence of the integrated upconversion intensity (area under $\mathrm{X}$ peak integrated from $2.054 \mathrm{eV}$ to $2.08 \mathrm{eV}$ ) on the energy difference $\mathrm{E}_{\mathrm{X}}-\mathrm{E}_{\mathrm{ex}}$ of the exciton $\mathrm{X}$ and the exciting photon energy $\mathrm{E}_{\mathrm{X}}$. At $7 \mathrm{~K}$ the energy gain of upconversion emission amounts to about $42 \mathrm{meV}$, which is comparable with the energy difference between the $\mathrm{X}$ and $\mathrm{T}_{\mathrm{S}}$ emission lines (spin singlet trion binding energy plus Fermi level energy ${ }^{21}$ ) and also nearly resonates with the energy of one optical phonon $\left(\mathrm{A}_{1}{ }_{1}\right.$ or $\left.\mathrm{E}^{\prime}\right)$. This suggests that at low temperature the upconversion photoluminescence process is related to the coupling between the trion (T) and exciton (X) states mediated by one optical phonon. Similarly to the work of Jones et al. ${ }^{11}$, we find that the higher energy triplet trion state $\left(\mathrm{T}_{\mathrm{T}}\right)$ peak dominates the upconversion emission (see Fig. 5b). Moreover, for the energy difference $\mathrm{E}_{\mathrm{X}}-\mathrm{E}_{\mathrm{ex}}$ between $32 \mathrm{meV}$ and $40 \mathrm{meV}$, the integrated upconversion intensity decreases with decreasing excitation photon energy, with the rate equal to $-0.079 \mathrm{meV}^{-1}$ (Fig. 5c), which is about three times higher (in absolute value) than the one estimated at $295 \mathrm{~K}$ under ambient condition.

We now turn to the analysing a mechanism responsible for the upconversion photoluminescence at intermediate temperatures. Figure 6a shows the PL spectrum detected at $70 \mathrm{~K}$. Coloured arrows indicate excitation energies of the upconversion photoluminescence spectra presented in Fig. 6b. At this temperature, the exciting photon energy required to achieve a detectable upconversion photoluminescence (gradual intensity growth just above the noise floor) is found to exceed $\mathrm{E}_{\mathrm{ex}}=2.009 \mathrm{eV}$. Following the upconversion photoluminescence spectra excited at different energies, presented in Fig. 6b, we see that with decreasing excitation energy from 2.036 to $2.026 \mathrm{eV}$, the intensity
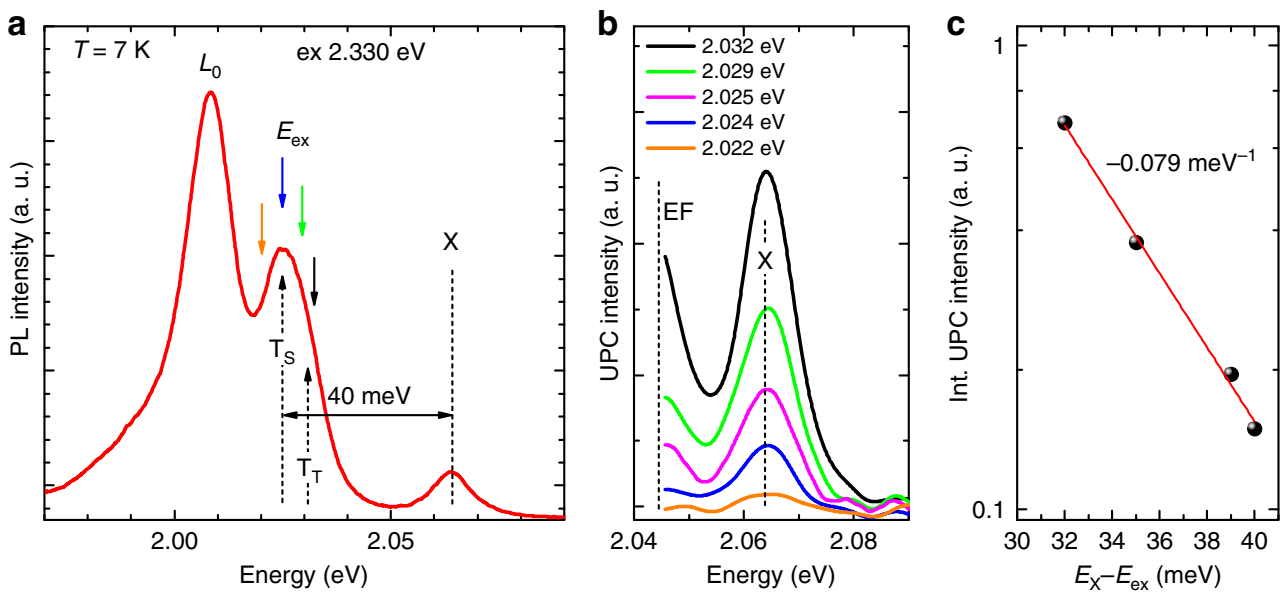

Fig. 5 Upconversion photoemission process in monolayer $\mathrm{WS}_{2}$ at $7 \mathrm{~K}$. a The PL spectrum recorded for $\mathrm{WS}_{2} / \mathrm{hBN}(136 \mathrm{~nm}) / \mathrm{SiO}_{2} / \mathrm{Si}$ at $7 \mathrm{~K}$. Black, green, blue and orange arrows point out excitation energies used in UPC experiment. $\mathbf{b}$ Examples of UPC spectra at $7 \mathrm{~K}$ excited with energies indicated by coloured arrows in a. c The dependence of the upconversion integrated intensity on the energy difference of the neutral exciton $(X)$ and the excitation photon energy $\left(E_{x}-E_{e x}\right)$ 
of the upconversion photoluminescence increases and achieves maximum at excitation with energy nearly equals to the energy of the trion in a spin singlet state $\mathrm{T}_{s}$ (green arrow in Fig. 6b). With further decreasing excitation energy, the upconversion photoluminescence decreases and is not detected at excitation energies below $2.009 \mathrm{eV}$. Figure $6 \mathrm{c}$ presents the dependence of the integrated intensity of upconversion photoluminescence on the energy difference $E_{X}-E_{e x}$ of the exciton $X$ and the exciting photon energy $E_{X}$. For the energy difference from $37 \mathrm{meV}$ to $60 \mathrm{meV}$, the integrated upconversion photoluminescence intensity decreases with decreasing excitation photon energy with the rate equal to $-0.054 \mathrm{meV}^{-1}$, which is about two times higher and about one and half times lower (in absolute value) than those estimated under ambient at 295 and $70 \mathrm{~K}$, respectively. Importantly, these results show that upconversion photoluminescence energy gain increases with increasing temperature. The energy gain of $\sim 60$ $\mathrm{meV}$ at $70 \mathrm{~K}$ suggests that more than one phonon is involved in the upconversion process.
To get further insight into the mechanism of photoluminescence at intermediate and high temperatures, we probe simultaneously the normal and upconversion photoluminescence spectra at temperatures from $20 \mathrm{~K}$ to $160 \mathrm{~K}$. At all temperatures, the excitation of the normal photoluminescence is fixed at 2.33 $\mathrm{eV}$, while the excitation energy of the upconversion photoluminescence is tuned in to maintain the constant energy separation between the excitation $\left(\mathrm{E}_{\mathrm{ex}}\right)$ and the exciton emission $\left(E_{X}\right)$. We have performed upconversion experiments for two different energy gains $\left(E_{X}-E_{e x}\right)$ equal to $37 \mathrm{meV}$ and $87 \mathrm{meV}$, respectively. Experiments are performed for two different $\mathrm{WS}_{2} /$ $\mathrm{hBN} / \mathrm{SiO}_{2} / \mathrm{Si}$ structures, which differ slightly in $\mathrm{X}$ emission intensity.

In Figure 7, the study of the normal photoluminescence and upconversion photoluminescence with the energy gain of $37 \mathrm{meV}$ at temperatures from $20 \mathrm{~K}$ to $160 \mathrm{~K}$ are presented. Figure $7 \mathrm{a}$ shows examples of the normal photoluminescence spectra at 20 $\mathrm{K}, 80 \mathrm{~K}, 100 \mathrm{~K}, 120 \mathrm{~K}, 140 \mathrm{~K}$, respectively. For each temperature,
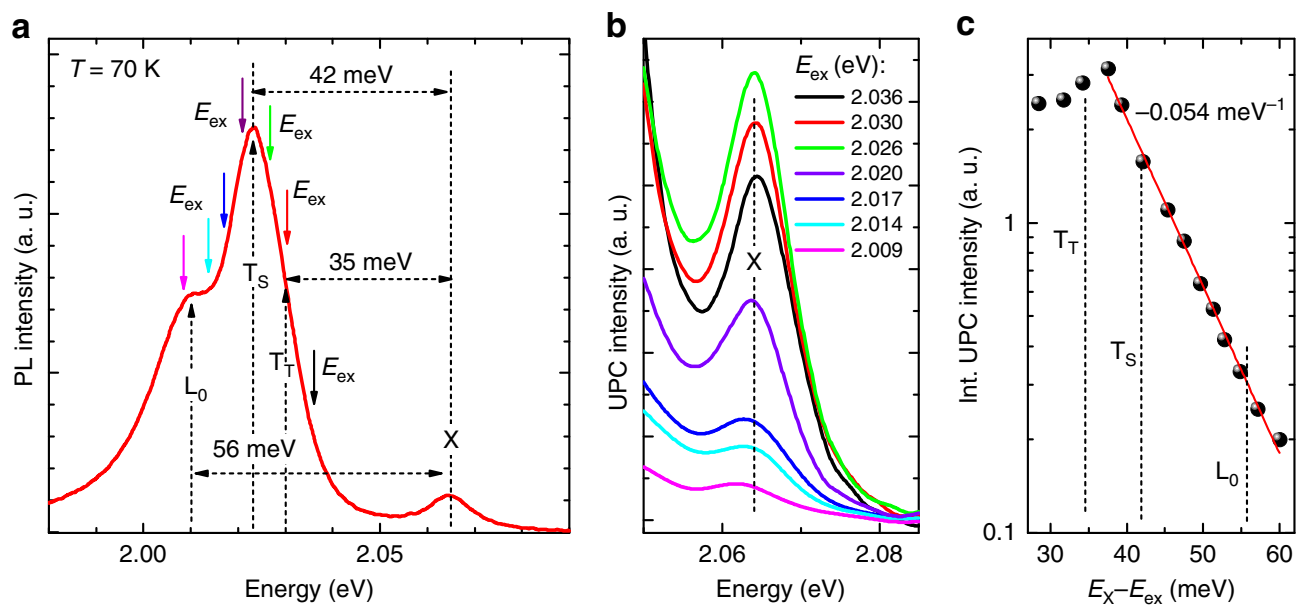

Fig. 6 Upconversion photoemission process in monolayer $\mathrm{WS}_{2}$ at $70 \mathrm{~K}$. a The PL spectrum recorded for $\mathrm{WS} / 2 / \mathrm{hBN}(136 \mathrm{~nm}) / \mathrm{SiO}_{2} / \mathrm{Si}$ at $70 \mathrm{~K}$. The coloured arrows point out excitation energies used in UPC experiment. $\mathbf{b}$ Examples of UPC spectra at $70 \mathrm{~K}$ excited with energies indicated by coloured arrows in $\mathbf{a}$. c The dependence of the upconversion integrated intensity on the energy difference of the neutral exciton $(X)$ and the excitation photon energy ( $\left.E_{X}-E_{e x}\right)$
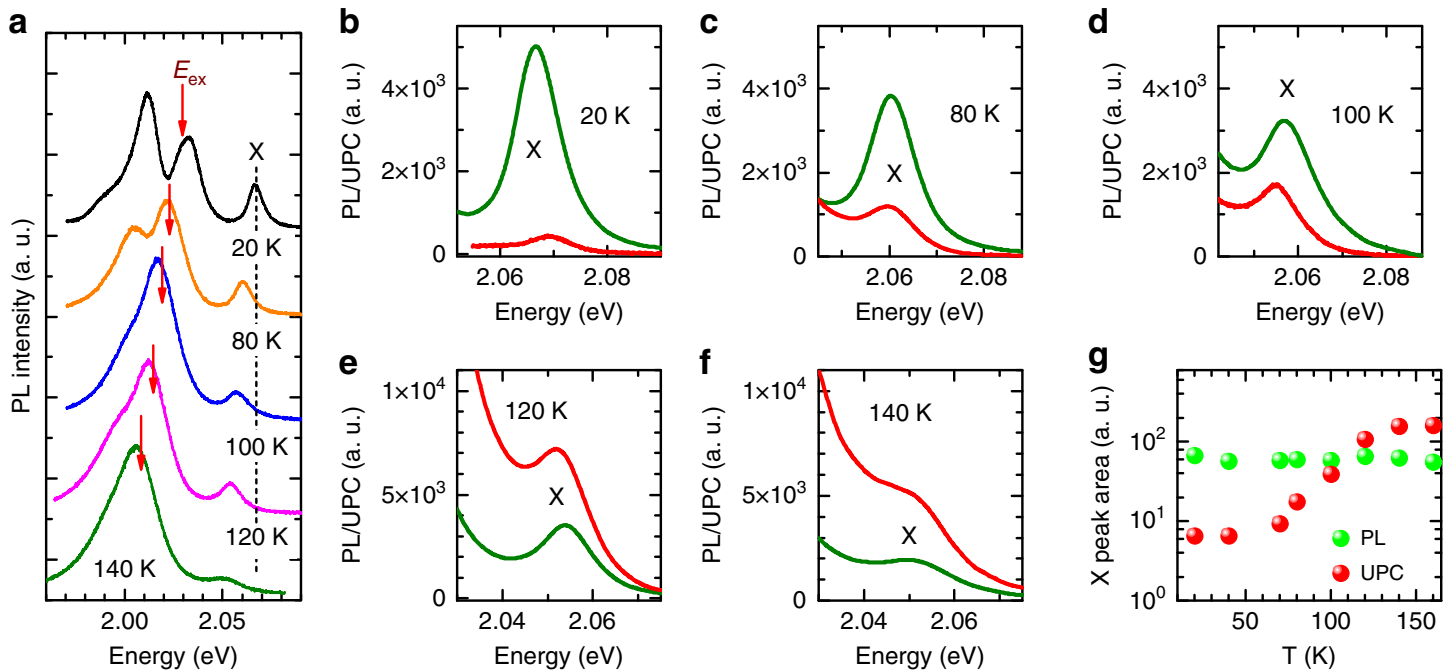

Fig. 7 Temperature dependence of upconverted emission with the energy gain of $37 \mathrm{meV}$. a Schematic representation of the experiment: typical PL spectra with indicated by red arrows excitation energies used in UPC measurements at corresponding temperatures. b-f Examples of normal (green line) and upconversion (red line) photoluminescence spectra (green and red lines, respectively) recorded at $20 \mathrm{~K}, 80 \mathrm{~K}, 100 \mathrm{~K}, 120 \mathrm{~K}, 140 \mathrm{~K}$, respectively. $\mathbf{g}$ The normal (green circles) and upconverted (red circles) excitonic photoluminescence integrated intensities as a function of temperature 
the excitation energy, marked by red arrows, is tuned in to maintain the constant $\mathrm{E}_{\mathrm{X}}-\mathrm{E}_{\mathrm{ex}}$ energy separation of $37 \mathrm{meV}$, which nearly matches the energy of the trion $(\mathrm{T})$ emission. Figure $7 \mathrm{~b}-\mathrm{f}$ compares the normal and upconversion photoluminescence spectra for $\mathrm{WS}_{2} / \mathrm{hBN}(136 \mathrm{~nm}) / \mathrm{SiO}_{2} / \mathrm{Si}$ structure at temperatures $20 \mathrm{~K}, 80 \mathrm{~K}, 100 \mathrm{~K}, 120 \mathrm{~K}, 140 \mathrm{~K}$, respectively. Figure $7 \mathrm{~g}$ shows the normal (green circles) and upconversion (red circles) photoluminescence integrated intensities of the neutral exciton $\mathrm{X}$ as a function of temperature (from $20 \mathrm{~K}$ to $160 \mathrm{~K}$ ). Both X emission intensities, at each temperature, are obtained by fitting of the PL/ UPC spectra by Lorentz function in common energy range indicated in Fig. 7b-f, respectively. The normal and upconversion photoluminescence intensities present different trends with respect to temperature: upconversion photoluminescence intensity increases and exceeds standard photoluminescence intensity at $120 \mathrm{~K}$, whereas normal photoluminescence remains nearly constant with increasing temperature. Among several investigated samples, both $\mathrm{WS}_{2} / \mathrm{SiO}_{2} / \mathrm{Si}$ and $\mathrm{WS}_{2} / \mathrm{hBN} / \mathrm{SiO}_{2} / \mathrm{Si}$, we find the same intensity trends of both the normal and upconversion photoluminescence. Here, the observed excitonic upconversion growth is governed by increasing phonon population ${ }^{10}$. It is also accompanied by simultaneous increase of the trion emission intensity with increasing temperature.

The study of the normal photoluminescence and upconversion photoluminescence with the energy gain of $87 \mathrm{meV}$ of the $\mathrm{WS}_{2} /$ $\mathrm{hBN}(115 \mathrm{~nm}) / \mathrm{SiO}_{2} / \mathrm{Si}$ structure at temperatures from $80 \mathrm{~K}$ to 160 $\mathrm{K}$ are presented in Fig. 8. The schematic representation of the experiment is shown in Fig. 8a, which presents examples of the normal photoluminescence spectra at $80 \mathrm{~K}, 100 \mathrm{~K}, 120 \mathrm{~K}, 140 \mathrm{~K}$, $160 \mathrm{~K}$, respectively. At each temperature, the excitation energy of the upconversion photoluminescence, indicated by red arrows, is tuned in to keep the constant energy gain $\left(\mathrm{E}_{\mathrm{X}}-\mathrm{E}_{\mathrm{ex}}\right)$ equal to 87 $\mathrm{meV}$, which corresponds to the energy of the states from the trion tail and slightly exceeds the energy of localized excitons L. Figure $8 \mathrm{~b}-\mathrm{f}$ compares the normal and upconversion photoluminescence spectra of $\mathrm{WS}_{2} / \mathrm{hBN}(115 \mathrm{~nm}) / \mathrm{SiO}_{2} / \mathrm{Si}$ structure at temperatures from $80 \mathrm{~K}$ to $160 \mathrm{~K}$, respectively. As in the experiment with the lower energy gain (Fig. $7 \mathrm{~g}$ ), we find consistent intensity increase of $\mathrm{X}$ upconversion photoluminescence and nearly constant normal $\mathrm{X}$ photoluminescence in temperature range from $100 \mathrm{~K}$ to $160 \mathrm{~K}$ (see Fig. $8 \mathrm{~g}$ ). However, for the higher energy gain $(87 \mathrm{meV})$, the intensity of $\mathrm{X}$ upconversion photoluminescence is relatively lower than the intensity of the normal $\mathrm{X}$ photoluminescence at each temperature. Here, the observed $\mathrm{H}$ excitonic upconversion growth at corresponding temperatures is again attributed to the increasing phonon population and is correlated with simultaneous increase of the trion emission. Additionally, this growth is accompanied by a gradual decrease of the localized excitons PL emission, also clearly observed in Fig. 8a or in Fig. 3e. These results indicate an efficient transfer between trion and exciton states mediated by resonant phonons. They also clearly confirm that in high temperature multi-phonon upconversion process the incident photon is rather in resonance with the states from the tail of the trion than in resonance with the exciton localized on the impurity.

The example of the optical contrast microscope image and atomic force microscope (AFM) imaging of the typical, studied $\mathrm{WS}_{2} / \mathrm{hBN} / \mathrm{SiO}_{2} / \mathrm{Si}$ heterostructure is presented in Fig. $9 \mathrm{a}-\mathrm{c}$.

Let us now discuss the efficiency of the upconversion photoluminescence process by means of the changes in the exciton emission intensity for different energy gains and gradual intensity descent rates. At $7 \mathrm{~K}$, we estimate a change in the $\mathrm{X}$ line intensity of 0.632 decade (see Fig. $5 c(40 \mathrm{meV}-32 \mathrm{meV}) \times 0.079$ $\mathrm{dec} / \mathrm{meV})$. Then, at $70 \mathrm{~K}$, we have a change in the $\mathrm{X}$ intensity of 1.2 decade (see Fig. $6 \mathrm{c}(60 \mathrm{meV}-37.5 \mathrm{meV}) \times 0.054)$, whereas at room temperature in ambient, we obtain a change in the $\mathrm{X}$ line intensity of 2.56 decade (see Fig. $2 b(150 \mathrm{meV}-50 \mathrm{meV}) \times$ 0.0256). This simple analysis shows that at $\mathrm{T}=295 \mathrm{~K}$ the upconversion photoluminescence intensity can be tuned by about two orders of magnitude over the mean energy gain of $100 \mathrm{meV}$.

\section{Discussion}

To summarise, we demonstrate here a room temperature prominent upconversion photoluminescence process in a monolayer semiconductor $\mathrm{WS}_{2}$, with the energy gain up to $150 \mathrm{meV}$. We identified this process as transitions between trions dressed by phonons and free exciton complexes. We also show that upconversion photoluminescence energy gain significantly depends on the temperature and increases from $42 \mathrm{meV}$ at $7 \mathrm{~K}$ to $150 \mathrm{meV}$ at $295 \mathrm{~K}$, indicating that high-temperature upconversion photoluminescence is indeed enabled by multi-phonon assisted transition. These results can be very promising for laser
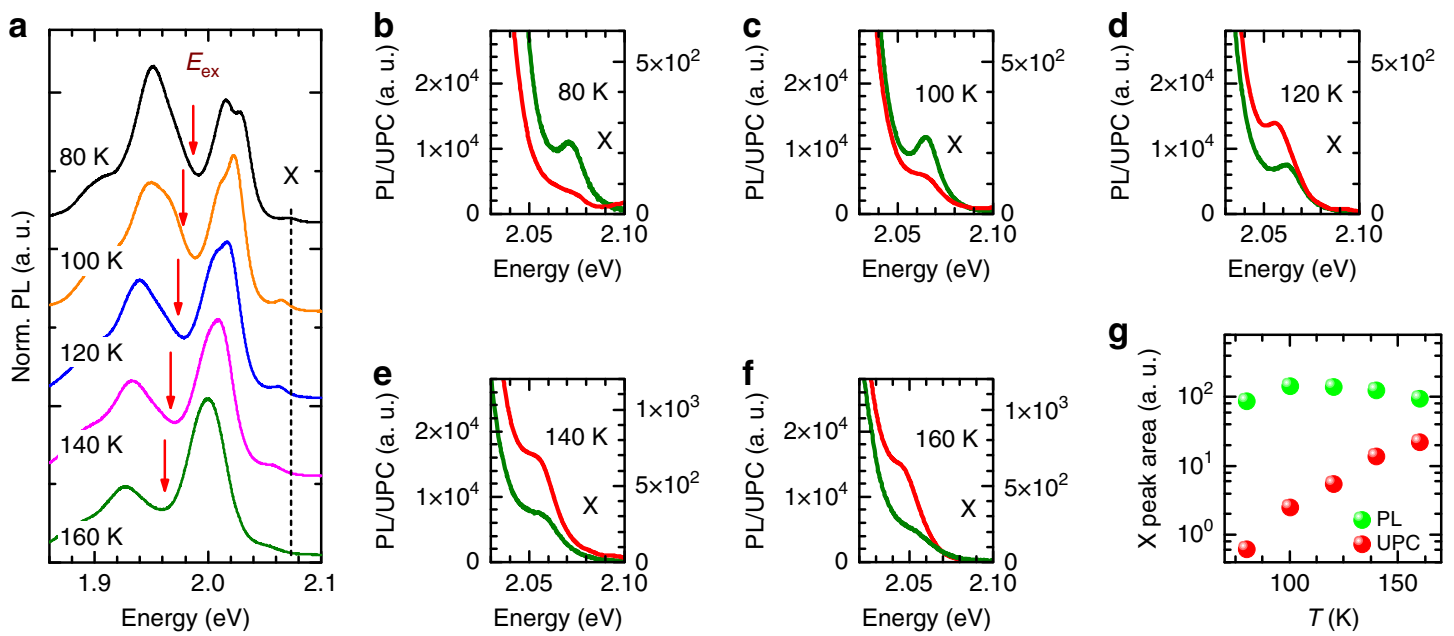

Fig. 8 Temperature dependence of upconverted emission with the energy gain of $87 \mathrm{meV}$. a Schematic representation of the experiment: typical PL spectra with indicated by red arrows excitation energies used in UPC measurements at corresponding temperatures. b-f Examples of normal (green line, left vertical scale) and upconversion (red line, right vertical scale) photoluminescence spectra recorded at $80 \mathrm{~K}, 100 \mathrm{~K}, 120 \mathrm{~K}, 140 \mathrm{~K}, 160 \mathrm{~K}$, respectively. $\mathbf{g}$ The normal (green circles) and upconverted (red circles) excitonic photoluminescence integrated intensities as a function of temperature from $80 \mathrm{~K}$ to $160 \mathrm{~K}$ 

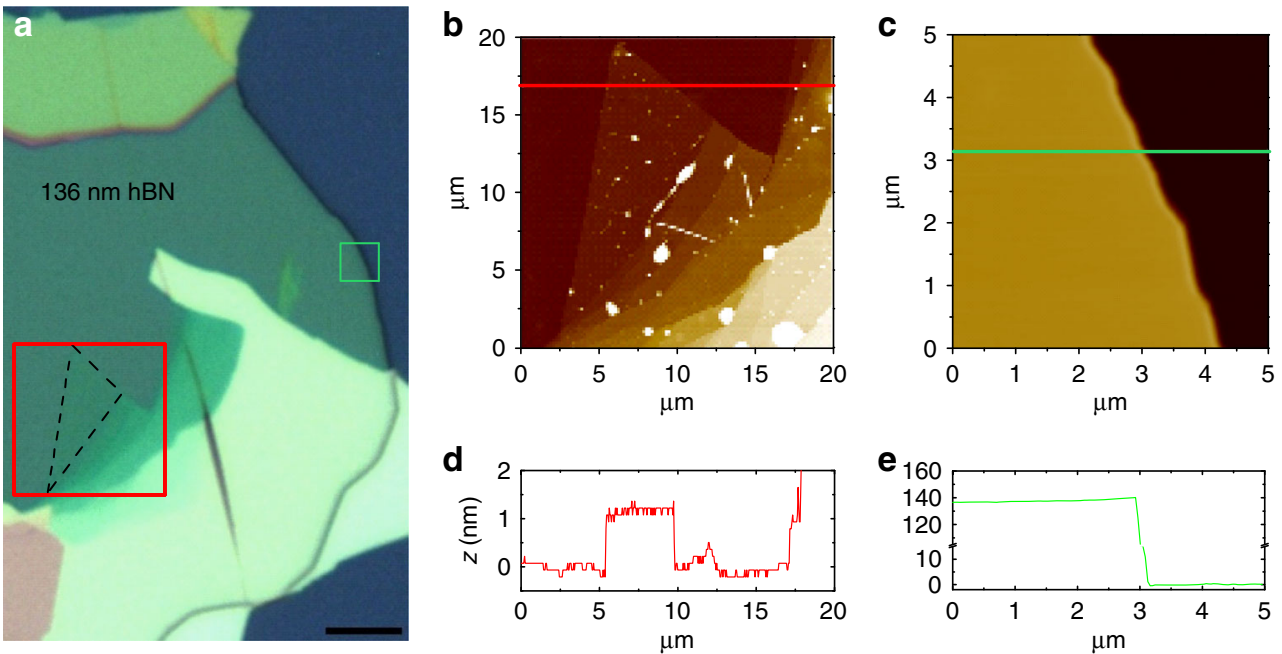

Fig. 9 Optical microscope contrast and AFM image of $\mathrm{WS}_{2} / \mathrm{hBN} / \mathrm{SiO}_{2} / \mathrm{Si}$ structure. a The optical microscope contrast image of WS $/ \mathrm{hBN} / \mathrm{SiO} 2 / \mathrm{Si}$ structure. The dashed black triangle shows the area corresponding to $\mathrm{WS}_{2}$ flake, whereas the red and green rectangles point out the areas corresponding to $\mathrm{WS}_{2} / \mathrm{hBN} / \mathrm{SiO}_{2} / \mathrm{Si}$ and $\mathrm{hBN} / \mathrm{SiO}_{2} / \mathrm{Si}$, respectively. $\mathbf{b}$ The AFM image of the area indicated by the red rectangle in $\mathbf{a}$. $\mathbf{c}$ The AFM image of the area indicated by the green rectangle in $\mathbf{a}$. $\mathbf{d}$ The height profile along the red line overlaid on the image in $\mathbf{b}$. $\mathbf{e}$ The height profile along the green line overlaid on the image in $\mathbf{c}$

refrigeration, energy harvesting and optoelectronics with atomically thin materials.

\section{Methods}

Samples preparation. The monolayers of $\mathrm{WS}_{2}$ studied here were prepared by mechanical exfoliation of bulk crystals grown by chemical vapour transport technique (CVT). Prior to the crystal growth, the powdered compound was prepared from the elements (W: $99.99 \%$; S: $99.999 \%$ ) by reaction at $T=1000{ }^{\circ} \mathrm{C}$ for 10 days in evacuated quartz ampoules. The chemical transport was achieved with $\mathrm{Br}_{2}$ as a transport agent in the amount of about $5 \mathrm{mg} / \mathrm{cm}^{3}$.

We prepared $\mathrm{WS}_{2} / \mathrm{hBN} / \mathrm{SiO}_{2} / \mathrm{Si}$ structures using hBN purchased from $2 \mathrm{D}$ Semiconductors. The $\mathrm{WS}_{2}$ crystals and hBN flakes with different thicknesses were mechanically exfoliated and then stacked using the deterministic all-dry stamping method, according to Castellanos-Gomez et al. ${ }^{46}$. We exfoliated hBN flake on a flexible PDMS Gel-Film stamp rigidly connected to a glass slide. Then the substrate and the stamp were placed under an optical microscope with XYZ stage attached. Application of long working distance objective enables us to locate and

deterministically transfer selected $\mathrm{hBN}$ flakes on the substrate by carefully bringing the stamp in contact with the substrate. The same procedure has been repeated for monolayer $\mathrm{WS}_{2}$, which has been deterministically transferred on hBN flake. With that method, we prepared monolayer $\mathrm{WS}_{2}$ on hBN flakes with different thicknesses on $\mathrm{Si}$ substrates with $300 \mathrm{~nm} \mathrm{SiO}_{2}$. Typical size of flakes exceeded $4 \times 4 \mu \mathrm{m}$. Monolayer character of $\mathrm{WS}_{2}$ flakes was determined by their different optical contrast and has been confirmed by Raman and PL measurements on PDMS (to ensure the same experimental conditions) and, after transfer on target substrate, by atomic force microscopy (AFM) imaging (Fig. 9a-e).

Experimental setup. The samples were mounted on a cold-finger of a nonvibrating closed circle cryostat, where temperature can be varied from 7 to $300 \mathrm{~K}$. Photoluminescence was excited either by single mode lasers: the second harmonic $532 \mathrm{~nm}$ line of Nd: YAG and $632.8 \mathrm{~nm}$ line of a He-Ne, or by $610-675 \mathrm{~nm}$ lines of a DCM dye laser. The laser beam was focused on the sample under normal incidence using a $50 \times$ high resolution, long distance microscope objective $(\mathrm{NA}=0.65)$. The diameter of excitation spot was equal to $\sim 1 \mu \mathrm{m}$. The spectra were analysed with a $0.5 \mathrm{~m}$ focal length spectrometer and a 1200 lines $/ \mathrm{mm}$ grating. A Peltiercooled Si charge couple device was used as a detector. To eliminate the laser scattering light and phosphorescence of dye laser a set of short and long pass razor edge filters was used. The Raman scattering measurements were performed in the same set-up in backscattering geometry. The reflectivity contrast spectra were measured also in the same set-up, with 600 lines/mm grating and a filament lamp used as a light source.

\section{Data availability}

The data that support the findings of this study are available from the corresponding author on reasonable request.
Received: 27 July 2018 Accepted: 7 December 2018

Published online: 10 January 2019

\section{References}

1. Epstein, R. I., Buchwald, M. I., Edwards, B. C., Gosnell, T. R. \& Mungan, C. E. Observation of laser-induced fluorescent cooling of a solid. Nature 377, 500-503 (1995).

2. Seletskiy, D. V. et al. Laser cooling of solids to cryogenic temperatures. Nat. Photonics 4, 161-164 (2010).

3. Seletskiy, D. V., Epstein, R. \& Sheik-Bahae, M. Laser cooling in solids: advances and prospects. Reports Prog. Phys. 79, 096401 (2016).

4. Finkeißen, E., Potemski, M., Wyder, P., Viña, L. \& Weimann, G. Cooling of a semiconductor by luminescence up-conversion. Appl. Phys. Lett. 75, 1258-1260 (1999)

5. Rupper, G., Kwong, N. H. \& Binder, R. Large excitonic enhancement of optical refrigeration in semiconductors. Phys. Rev. Lett. 97, 1-4 (2006).

6. Eshlaghi, S., Worthoff, W., Wieck, A. D. \& Suter, D. Luminescence upconversion in GaAs quantum wells. Phys. Rev. B 77, 1-7 (2008).

7. Khurgin, J. B. Role of bandtail states in laser cooling of semiconductors. Phys. Rev. B 77, 1-9 (2008).

8. Zhang, J., Li, D., Chen, R. \& Xiong, Q. Laser cooling of a semiconductor by 40 kelvin. Nature 493, 504-508 (2013).

9. Akizuki, N., Aota, S., Mouri, S., Matsuda, K. \& Miyauchi, Y. Efficient nearinfrared up-conversion photoluminescence in carbon nanotubes. Nat. Commun. 6, 1-6 (2015).

10. Jones, A. M. et al. Excitonic luminescence upconversion in a two-dimensional semiconductor. Nat. Phys. 12, 323-327 (2016).

11. Manca, M. et al. Enabling Valley selective exciton scattering in monolayer $\mathrm{WSe}_{2}$ through upconversion. Nat. Commun. 8, 1-7 (2017).

12. Mak, K. F., Lee, C., Hone, J., Shan, J. \& Heinz, T. F. Atomically thin $\mathrm{MoS}_{2}$ : a new direct-gap semiconductor. Phys. Rev. Lett. 105, 136805 (2010).

13. Splendiani, A. et al. Emerging photoluminescence in monolayer $\mathrm{MoS}_{2}$. Nano. Lett. 10, 1271-1275 (2010).

14. Jadczak, J., Delgado, A., Bryja, L., Huang, Y. S. \& Hawrylak, P. Robust hightemperature trion emission in monolayers of $\mathrm{Mo}\left(\mathrm{S}_{\mathrm{y}} \mathrm{Se}_{1-\mathrm{y}}\right)_{2}$ alloys. Phys. Rev. B 95, 195427 (2017)

15. Bieniek, M. et al. Band nesting, massive Dirac fermions, and valley Landé and Zeeman effects in transition metal dichalcogenides: a tight-binding model. Phys. Rev. B 97, 1-9 (2018).

16. Mitioglu, A. A. et al. Optical manipulation of the exciton charge state in single-layer tungsten disulfide. Phys. Rev. B 88, 245403 (2013).

17. Ye, Z. et al. Probing excitonic dark states in single-layer tungsten disulphide. Nature 513, 214-218 (2014)

18. Chernikov, A. et al. Exciton binding energy and nonhydrogenic Rydberg series in monolayer $\mathrm{WS}_{2}$. Phys. Rev. Lett. 113, 076802 (2014). 
19. Plechinger, G. et al. Trion fine structure and coupled spin-valley dynamics in monolayer tungsten disulfide. Nat. Commun. 7, 12715 (2016).

20. Molas, M. R. et al. The optical response of monolayer, few-layer and bulk tungsten disulfide. Nanoscale 9, 13128-13141 (2017).

21. Jadczak, J., Kutrowska-Girzycka, J., Kapuściński, P., Wójs, A. \& Bryja, L. Probing of free and localized excitons and trions in atomically thin $\mathrm{WSe}_{2}, \mathrm{WS}_{2}$, $\mathrm{MoSe}_{2}$ and $\mathrm{MoS}_{2}$ in photoluminescence and reflectivity experiments. Nanotechnology 28, 395702 (2017).

22. Tongay, S. et al. Broad-range modulation of light emission in two-dimensional semiconductors by molecular physisorption gating. Nano. Lett. 13, 2831-2836 (2013).

23. Miller, B., Parzinger, E., Vernickel, A., Holleitner, A. W. \& Wurstbauer, U. Photogating of mono- and few-layer $\mathrm{MoS}_{2}$. Appl. Phys. Lett. 106, 122103 (2015).

24. Radisavljevic, B., Radenovic, A., Brivio, J., Giacometti, V. \& Kis, A. Singlelayer $\mathrm{MoS}_{2}$ transistors. Nat. Nanotechnol. 6, 147-150 (2011).

25. Shang, J. et al. Revealing electronic nature of broad bound exciton bands in twodimensional semiconducting $\mathrm{WS}_{2}$ and $\mathrm{MoS}_{2}$. Phys. Rev. Mater. 1, 074001 (2017).

26. Kadantsev, E. S. \& Hawrylak, P. Electronic structure of a single $\mathrm{MoS}_{2}$ monolayer. Solid State Commun. 152, 909-913 (2012).

27. Kioseoglou, G. et al. Valley polarization and intervalley scattering in monolayer $\mathrm{MoS}_{2}$. Appl. Phys. Lett. 101, 221907 (2012).

28. Mak, K. F., He, K., Shan, J. \& Heinz, T. F. Control of valley polarization in monolayer $\mathrm{MoS}_{2}$ by optical helicity. Nat. Nanotechnol. 7, 494-498 (2012).

29. Geim, A. K. \& Grigorieva, I. V. Van der Waals heterostructures. Nature 499, 419-425 (2013).

30. Ross, J. S. et al. Electrical control of neutral and charged excitons in a monolayer semiconductor. Nat. Commun. 4, 1474 (2013).

31. Xu, X., Yao, W., Xiao, D. \& Heinz, T. F. Spin and pseudospins in layered transition metal dichalcogenides. Nat. Phys. 10, 343-350 (2014).

32. Scrace, T. et al. Magnetoluminescence and valley polarized state of a twodimensional electron gas in $\mathrm{WS}_{2}$ monolayers. Nat. Nanotechnol. 10, 603-607 (2015).

33. Schneider, L. M. et al. The influence of the environment on monolayer tungsten diselenide photoluminescence. Nano-Struct. \& Nano-Objects 15, 84-97 (2018).

34. Tuan, D. V., Jones, A. M., Yang, M., Xu, X. and Dery, H., Virtual trions in the photoluminescence of monolayer transition-metal dichalcogenides. Preprint at https://arxiv.org/abs/1805.08722 (2018).

35. Chen, S. Y., Zheng, C., Fuhrer, M. S. \& Yan, J. Helicity-resolved Raman scattering of $\mathrm{MoS}_{2}, \mathrm{MoSe}_{2}, \mathrm{WS}_{2}$, and WSe $\mathrm{W}_{2}$ atomic layers. Nano. Lett. 15, 2526-2532 (2015).

36. Molina-Sánchez, A. \& Wirtz, L. Phonons in single-layer and few-layer $\mathrm{MoS}_{2}$ and $\mathrm{WS}_{2}$. Phys. Rev. B 84, 1-8 (2011).

37. Yang, J., Lee, J. U. \& Cheong, H. Excitation energy dependence of Raman spectra of few-layer $\mathrm{WS}_{2}$. FlatChem 3, 64-70 (2017).

38. Carvalho, B. R., Malard, L. M., Alves, J. M., Fantini, C. \& Pimenta, M. A. Symmetry-Dependent Exciton-Phonon Coupling in $2 \mathrm{D}$ and Bulk $\mathrm{MoS}_{2}$ Observed by Resonance Raman Scattering. Phys. Rev. Lett. 114, 136403 (2015).

39. Chakraborty, B. et al. Symmetry-dependent phonon renormalization in monolayer $\mathrm{MoS}_{2}$ transistor. Phys. Rev. B 85, 2-5 (2012).

40. Molas, M. R., Nogajewski, K., Potemski, M. \& Babiński, A. Raman scattering excitation spectroscopy of monolayer $\mathrm{WS}_{2}$. Sci. Rep. 7, 5036 (2017).

41. Jiang, Z. X., McCombe, B. D. \& Hawrylak, P. Donor impurities as a probe of electron correlations in a two-dimensional electron gas in high magnetic fields. Phys. Rev. Lett. 81, 3499-3502 (1998).
42. Wang, G. et al. Giant enhancement of the optical second-harmonic emission of $\mathrm{WSe}_{2}$ monolayers by laser excitation at exciton resonances. Phys. Rev. Lett. 114, 1-6 (2015)

43. Seyler, K. L. Electrical control of second-harmonic generation in a $\mathrm{WSe}_{2}$ monolayer transistor. Nat. Nanotechnol. 10, 1-5 (2015).

44. Kumar, N. et al. Exciton-exciton annihilation in $\mathrm{MoSe}_{2}$ monolayers. Phys. Rev B 89, 125427 (2014)

45. Mouri, S. et al. Nonlinear photoluminescence in atomically thin layered $\mathrm{WSe}_{2}$ arising from diffusion-assisted exciton-exciton annihilation. Phys. Rev. B 90, 155449 (2014).

46. Castellanos-Gomez, A. et al. Deterministic transfer of two-dimensional materials by all-dry viscoelastic stamping. 2D Mater. 1, 011002 (2014).

\section{Acknowledgements}

J.J. and J.K.-G. acknowledge support by the Polish NCN Grant "Beethoven" No. 2016/23/ G/ST3/04114. L.B. acknowledges support by the Polish NCN Grant 2013/09/B/ST3/ 02528. P.H. and M.B. acknowledge support by the University of Ottawa Research Chair in Quantum Theory of Materials.

\section{Author contributions}

J.J. and L.B. designed and supervised the experiments. J.J. performed the measurements, analysed and interpreted the data with discussion from L.B. Y.-S.H. synthesized the $\mathrm{WS}_{2}$ single crystals by the CVT method. J.K.-G. prepared and characterized $\mathrm{WS}_{2}$ monolayers J.K.-G., P.K. and M.B. performed characterization of the bulk WS $W_{2}$. J.J., L.B. and P.H. wrote the paper. M.B. acknowledges financial support from National Science Center (NCN), Poland, grant Maestro No. 2014/14/A/ST3/00654.

\section{Additional information}

Competing interests: The authors declare no competing interests.

Reprints and permission information is available online at http://npg.nature.com/ reprintsandpermissions/

Journal peer review information: Nature Communications thanks the anonymous reviewers for their contributions to the peer review of this work. Peer Reviewer Reports are available.

Publisher's note: Springer Nature remains neutral with regard to jurisdictional claims in published maps and institutional affiliations.

(cc) (i) Open Access This article is licensed under a Creative Commons Attribution 4.0 International License, which permits use, sharing, adaptation, distribution and reproduction in any medium or format, as long as you give appropriate credit to the original author(s) and the source, provide a link to the Creative Commons license, and indicate if changes were made. The images or other third party material in this article are included in the article's Creative Commons license, unless indicated otherwise in a credit line to the material. If material is not included in the article's Creative Commons license and your intended use is not permitted by statutory regulation or exceeds the permitted use, you will need to obtain permission directly from the copyright holder. To view a copy of this license, visit http://creativecommons.org/ licenses/by/4.0/.

(c) The Author(s) 2019 